\title{
MULTI-OBJECTIVE OPTIMIZATION OF ALUMINIUM FOAM MANUFACTURING PARAMETERS
}

\author{
Surace, R.*; Bruno, S. ; De Filippis, L. A. C. ${ }^{* *} \&$ Ludovico, A. D. \\ * Politecnico di Bari, Dipartimento di Ingegneria Meccanica e Gestionale, viale Japigia 182, Bari, Italy \\ ${ }^{* *}$ Politecnico di Bari, Dipartimento di Ingegneria dell’Ambiente e per lo Sviluppo Sostenibile, \\ viale del Turismo 8, Taranto, Italy \\ E-Mail: r.surace@poliba.it, simonebruno@infinito.it, ludovico@poliba.it, l.defilippis@poliba.it
}

\begin{abstract}
Aluminium foams are a new class of materials with low densities, large specific surface and novel physical and mechanical properties. Their applications are extremely varied: for light weight structural components, for filters and electrodes and for shock or sound absorbing products. Recently, interesting foaming technology developments have proposed metallic foams as a valid commercial chance; foam manufacturing techniques include solid, liquid or vapour state methods. The foams presented in this study are produced by Melt Gas Injection (MGI) process starting from melt aluminium. The objective of this paper is to develop a method for the analysis of the effects of process parameters on the quality of foam parts and to determine their optimal combination. The effects of the foaming parameters are studied by the Taguchi method, applied to design an orthogonal experimental array. A multi-objective optimization approach is then proposed by simultaneously minimizing the relative density and maximizing the absorbed energy efficiency.

(Received in July 2008, accepted in December 2008. This paper was with the authors 2 months for 1 revision.)
\end{abstract}

Key Words: Aluminium Foam, Melt Gas Injection Process, Taguchi Method, MultiObjective Optimization

\section{INTRODUCTION}

Metal foams are relatively new materials which have attracted the increasing attention of industries, especially of automotive industry, around the world for their novel physical and mechanical properties. A disadvantage of metal foams is the inhomogeneity due to the stochastic nature of manufacturing processes. However the goal of production is not to achieve necessarily homogeneous structure, but predictable properties in a reproducible way. Thus, in order to obtain the desired parts it is necessary to study the influence of foaming parameters.

The melt-foaming process is a continuous, gas injection method developed simultaneously and independently by Alcan [1] and Norsk Hydro [2] in the late 1990s and it is called Melt Gas Injection (MGI). The starting material, usually a metal matrix composite, is molten with conventional foundry equipment and transferred to a tundish where gas is injected in it via a nozzle incorporated into a rotating impeller, thus forming a dispersion of small bubbles. Fig. 1 shows a sketch of the process [2]. The reinforcing particles are typically $\mathrm{SiC}$ or $\mathrm{Al}_{2} \mathrm{O}_{3}$, the volume fraction ranges from 10 to $20 \%$ and the mean particle size from 5 to $20 \mathrm{~mm}$. The ceramic particles trap gas bubbles owing to the favorable interface energy and serve as stabilizer of the cell walls and delay their coalescence. They also reduce the velocity of the rising bubbles by increasing the viscosity of the melt and the relative stabilising mechanisms are discussed in literature [3-5]. The bubbles size can be controlled by adjusting the process parameters: the gas flow rate, the propeller design (number of nozzles and their 
size), and its spin speed. To select the foaming parameters properly different approaches have been proposed based on wide experimental effort with high cost in terms of time and materials [6-8].

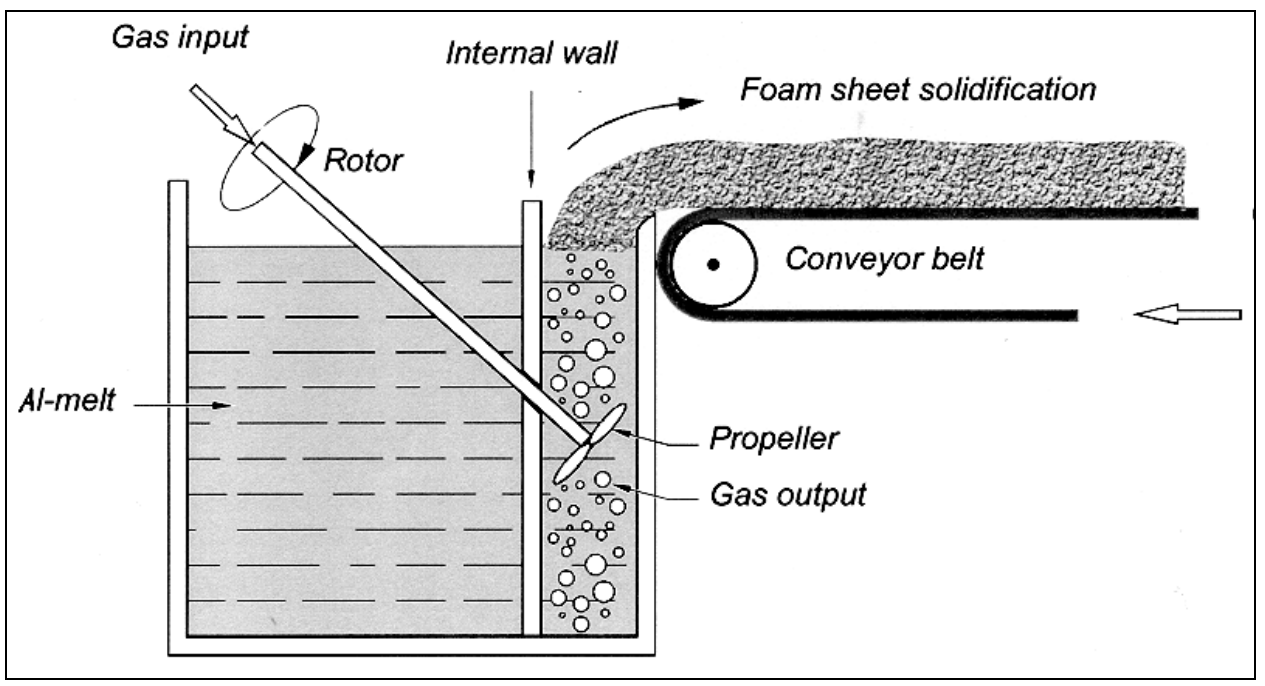

Figure 1: Apparatus for MGI [2].

In this paper an alternative approach based on the Taguchi method [9] is used to determine the parameters more efficiently. Taguchi proposed that the engineering optimization of a process should be carried out in three step approach: the system design, the parameter design and the tolerance design. In the parameters design Taguchi employs an orthogonal arrays to study the whole parameters space with only a small number of experiments. A loss function is then defined to calculate the deviation between the experimental value and the desired value. The value of the loss function is further transformed into a signal-to-noise $(\mathrm{S} / \mathrm{N})$ ratio that can be calculated considering three performance characteristics: the lower-the-better, the higher-the-better, and the nominal-the-best [10]. The greater is the $\mathrm{S} / \mathrm{N}$ ratio, the smaller is the variance of considered parameter around the desired value and the better is the performance characteristic. Taguchi method has been already applied in different industrial processes and operations: turning [10-11], milling [12], casting [13], welding [14], metal forming [15], and rapid prototyping [16]. No applications have been found in the field of material processing especially for metal foam manufacturing.

The Taguchi method can optimize qualitative characteristics through the setting of design parameters, and can reduce the sensitivity of the system performance to sources of variation of a single characteristic [11-13]. If more than one characteristic is simultaneously considered for the same process, the method may not give a unique optimal combination of parameters, especially if these characteristics compete with each other. Thus, the attempting to optimize more than one objective makes the problem a multi-objective one [17]. The common multiobjective solution methods are: the weighted sum method, the $\varepsilon$-constraint method and the goal attainment method [18]. The goal attainment method, used in this study, provides a convenient intuitive interpretation of the design problem which is solvable using standard optimization procedures; there is always at least one Pareto optimal solution, also called the nondominated solution, which balances the objectives in a unique and optimal way. In this paper a multi-objective optimization approach is proposed by integrating the classical mathematical method with the Taguchi method, and the optimization problem of aluminium foam process parameters is solved by the goal attainment method. The steps of optimization of this study are presented in Fig. 2. 


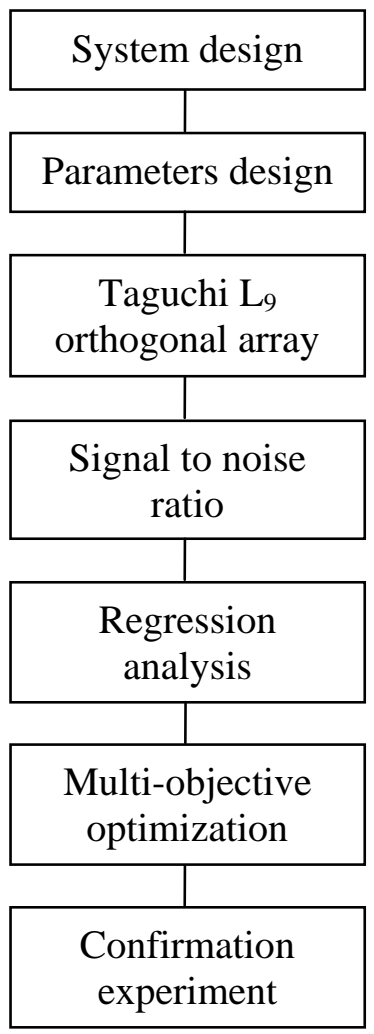

Figure 2: Steps of the optimization process.

\section{METHODOLOGY AND RESULTS}

\subsection{Selection of parameters and orthogonal array construction}

Only a few studies on MGI method are nowadays available and the knowledge on correlations between the final properties of the foams and the process parameters is still not complete. The process control for aluminium foam is regarded to be difficult due to its multi-variability and invisibility.

In this investigation, the aluminium foam has been produced by melting aluminium ingots in a graphite crucible by an induction furnace. The applied foaming equipment has been specifically designed and optimized for these experiments. It is formed by a rotating system that allows distributing the bubbles in the melt. The experimental apparatus is constituted by a drill, a shaft, a propeller, a panel of stainless steel, and a pipe for the gas injection. This system is designed in a proper way that allows the gas coming out from the pipe, and diffusing homogenously in the bath.

Duralcan metal matrix composite (A356/SiC/20p) with a particle size of approximately 12 $\mu \mathrm{m}$ is used. Aluminium 356 (called also AlSi7) has the following composition: Al 90.1-93.3 wt \%, Si 6.5-7.5 wt \%, Mg 0.2-0.45 wt \%, Cu max 0.25 \%, Fe max 0.6 \%, Mn max 0.35\%.

Nitrogen is injected as foaming medium and temperature has been kept at $700{ }^{\circ} \mathrm{C}$. The process is governed by controlling the pressure and flow rate of the blowing gas. After screening using different combinations of parameters and obtaining different results, the factors selected for observation are: flow rate $(Q)$, spin speed of the propeller $(N)$, and silicon carbide particles fraction $(F)$, because they show the high influence on foam properties (mechanical and morphological) and three levels are chosen for each of them (Table I). A L9 orthogonal array is selected as shown in Table II. Fig. 3 shows the foam samples obtained with parameters of different runs. 
Table I: Levels of foaming parameters.

\begin{tabular}{|c|c|c|c|c|}
\hline Symbol & Parameter & Level 1 & Level 2 & Level 3 \\
\hline$Q$ & Gas flow rate (l/min) & 2 & 4 & 8 \\
\hline$N$ & Spin speed (RPM) & 300 & 700 & 900 \\
\hline$F$ & SiC fraction (wt \%) & 10 & 15 & 20 \\
\hline
\end{tabular}

Table II: A L9 Taguchi orthogonal design.

\begin{tabular}{|c|c|c|c|}
\hline \multirow{2}{*}{ Run no. } & \multicolumn{3}{|c|}{ Parameters level } \\
\cline { 2 - 4 } & $\boldsymbol{Q}$ & $\boldsymbol{N}$ & $\boldsymbol{F}$ \\
\hline 1 & 1 & 1 & 1 \\
\hline 2 & 1 & 2 & 2 \\
\hline 3 & 1 & 3 & 3 \\
\hline 4 & 2 & 1 & 2 \\
\hline 5 & 2 & 2 & 3 \\
\hline 6 & 2 & 3 & 1 \\
\hline 7 & 3 & 1 & 3 \\
\hline 8 & 3 & 2 & 1 \\
\hline 9 & 3 & 3 & 2 \\
\hline
\end{tabular}
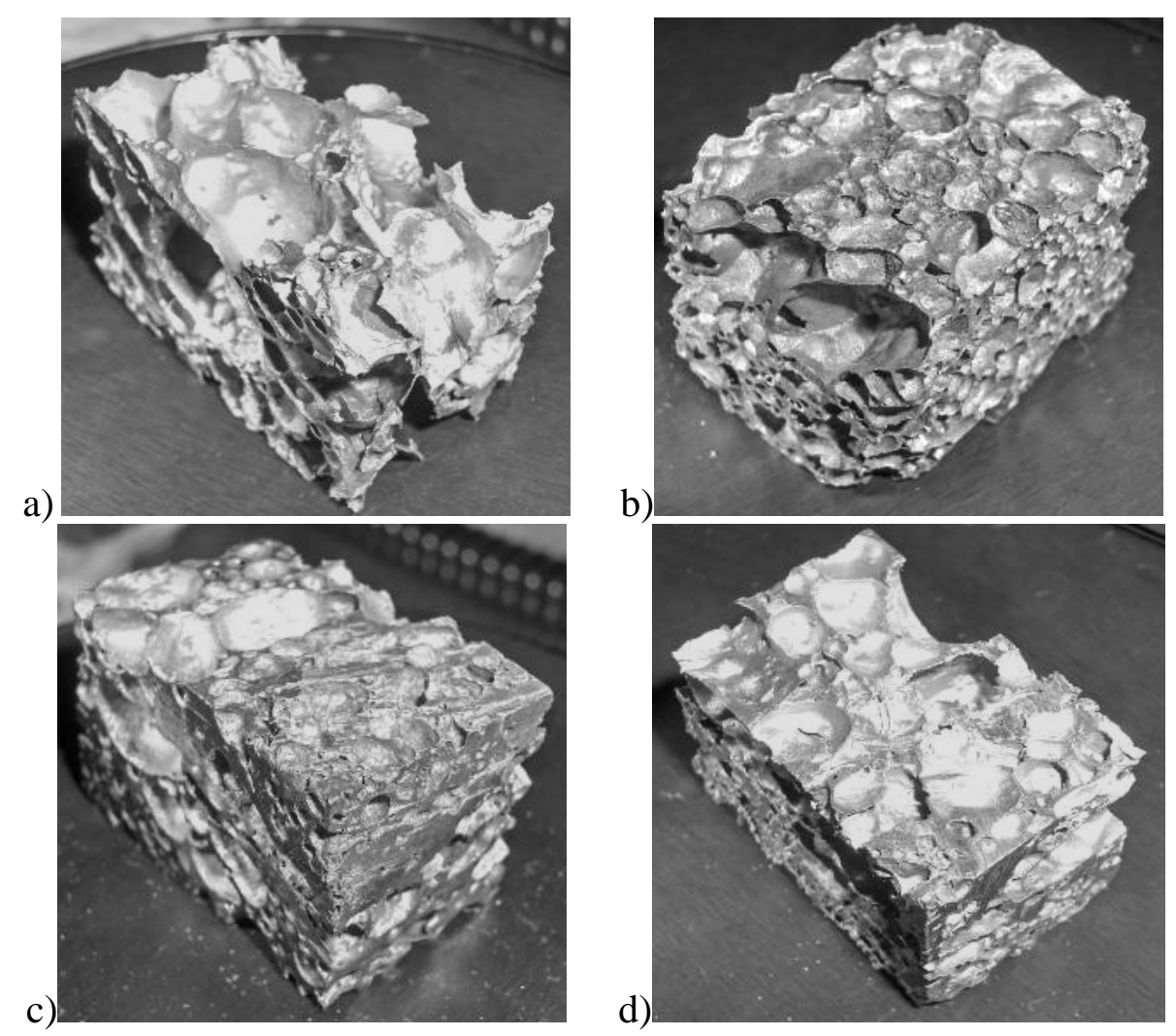

Figure 3: Samples obtained from different runs: a) no. 2 (Q1, N2, F2), b) no. 5 (Q2, N2, F3), c) no. $7(Q 3, N 1, F 3)$ and d) no. $9(Q 3, N 3, F 2)$.

\subsection{Objective functions}

To study the produced aluminium foam, a morphological and mechanical characterization has been carried out. The obtained samples were longitudinally cut with metallographic saw to 
minimize the cells damage (specimen dimensions $20 \times 20 \times 30 \mathrm{~mm}^{3}$ ); owing to the high content of ceramic particles, machining of metal matrix composite (MMC) foams is often difficult.

In aluminium foam manufacturing the primary objective is to get the relative density lower as possible without any failure happening. Relative density is defined as the ratio of the foam density and the bulk metal density (that is different for the three used alloy with different fraction of $\mathrm{SiC}$ ):

$$
R D=\frac{\rho_{F}}{\rho M}
$$

where: $\rho_{F}$ is the foam density and $\rho_{M}$ is the bulk metal density.

It is calculated for each specimen (Fig. 3) measuring the sample weights by an analytical balance and physical dimensions.

Energy absorption efficiency is also fundamental for metal foam applications especially for weight reduction in transport industries and it should be as high as possible. The energy absorption capacity of foam is defined as the maximum energy that can be dissipated by a unit mass of foam. The absorbed energy per unit volume, in a certain strain interval, is equal to the area below the stress-strain curve (eq. 2), and the efficiency of energy absorption $\eta$, is the ratio between the absorbed energy of the real material and the absorbed energy of an ideal absorber that is an ideal plastic material (eq. 3) given by the product of the maximum force applied over that collapse distance and the specimen length [19]. For high efficiency, little or no slope in the plateau region is desired. A large value for densification strain is also desirable, in order to increase the total energy absorbed by the foam.

$$
\begin{gathered}
E=\int_{0}^{\varepsilon_{D}} \sigma(\varepsilon) \cdot d \varepsilon \\
\eta=\frac{\int_{0}^{\varepsilon_{D}} \sigma(\varepsilon) \cdot d \varepsilon}{E_{\max }(\varepsilon) \cdot \varepsilon}
\end{gathered}
$$

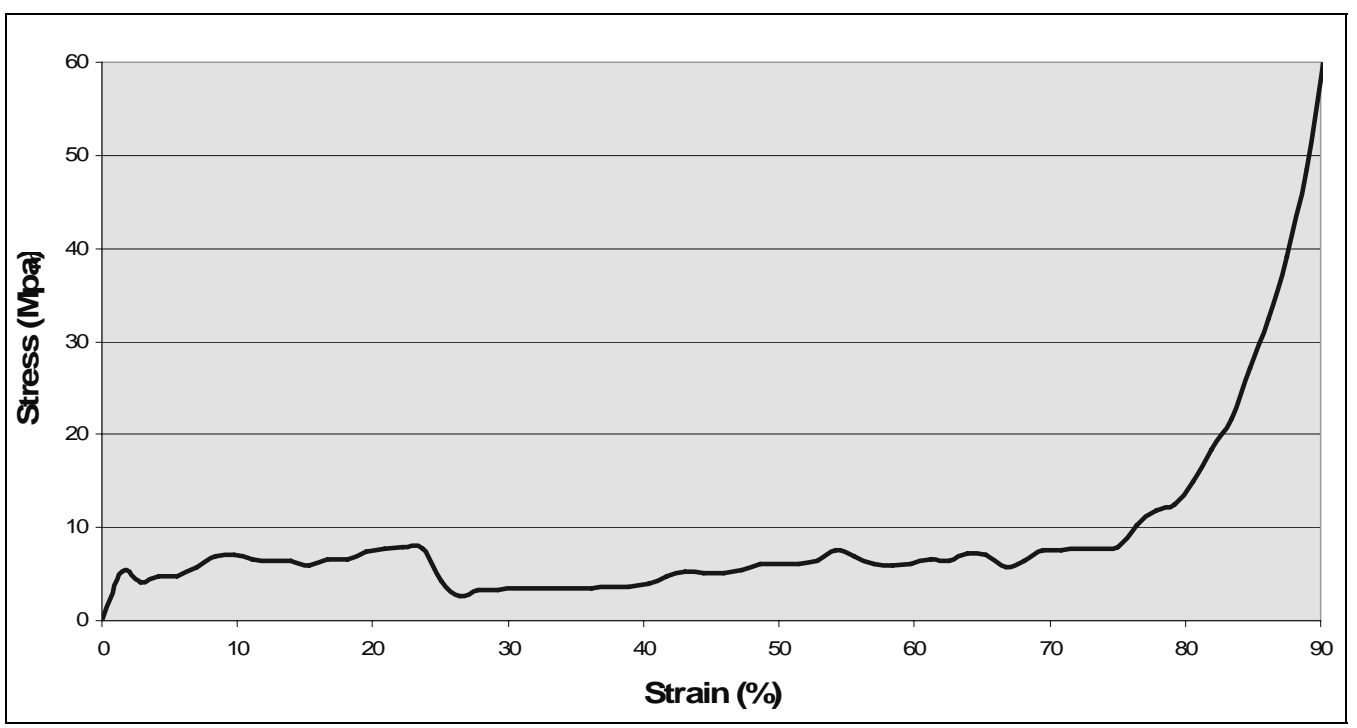

Figure 4: Stress-strain curve for the sample no. 5 (Q2, N2, F3).

In this investigation, the energy absorption efficiencies are calculated at $50 \%$ of deformation from stress-strain curves. The curves has been obtained (by machine model 
INSTRON 5869) following the statements of the standard test method for compressive properties of metal foam prepared by Cymat Company [20] taking into account ASTM and DIN standards. Flat surfaces of foam specimens have been prepared by machining, even if it has not been possible to avoid some cell walls ruptures. The energy efficiency is defined by the ratio of the energy absorbed after a fixed collapse (in this case $50 \%$ deformation) to the ideal energy absorption. Fig. 4 shows stress-strain curve for the sample no. 5.

The two objectives relative density and energy efficiency may conflict in the process since high energy absorption efficiency is strictly correlated with high relative density and vice versa.

\subsection{Analysis of the $\mathrm{S} / \mathrm{N}$ ratios}

After conducting the experimentation and applying Taguchi analysis, the results of relative density, energy efficiency and the relatively $\mathrm{S} / \mathrm{N}$ ratios are shown in Table III. To obtain optimal foaming performance, the lower-the-better performance characteristic has been taken for relative density and the higher-the-better for energy efficiency. The average $\mathrm{S} / \mathrm{N}$ ratios of both parameters are shown in Fig. 5.

Table III: Experimental results for relative density, energy efficiency and S/N ratios.

\begin{tabular}{|c|c|c|c|c|}
\hline Run no. & Relative density & S/N ratio & Energy efficiency & S/N ratio \\
\hline 1 & 0.301 & 10.429 & 0.5600 & -5.036 \\
\hline 2 & 0.079 & 22.047 & 0.9160 & -0.762 \\
\hline 3 & 0.180 & 14.895 & 0.8444 & -1.469 \\
\hline 4 & 0.242 & 12.324 & 0.8200 & -1.724 \\
\hline 5 & 0.174 & 15.189 & 0.8366 & -1.550 \\
\hline 6 & 0.182 & 14.799 & 0.9723 & -0.244 \\
\hline 7 & 0.240 & 12.396 & 0.6887 & -3.239 \\
\hline 8 & 0.210 & 13.556 & 0.4955 & -6.099 \\
\hline 9 & 0.154 & 16.250 & 0.4935 & -6.134 \\
\hline
\end{tabular}

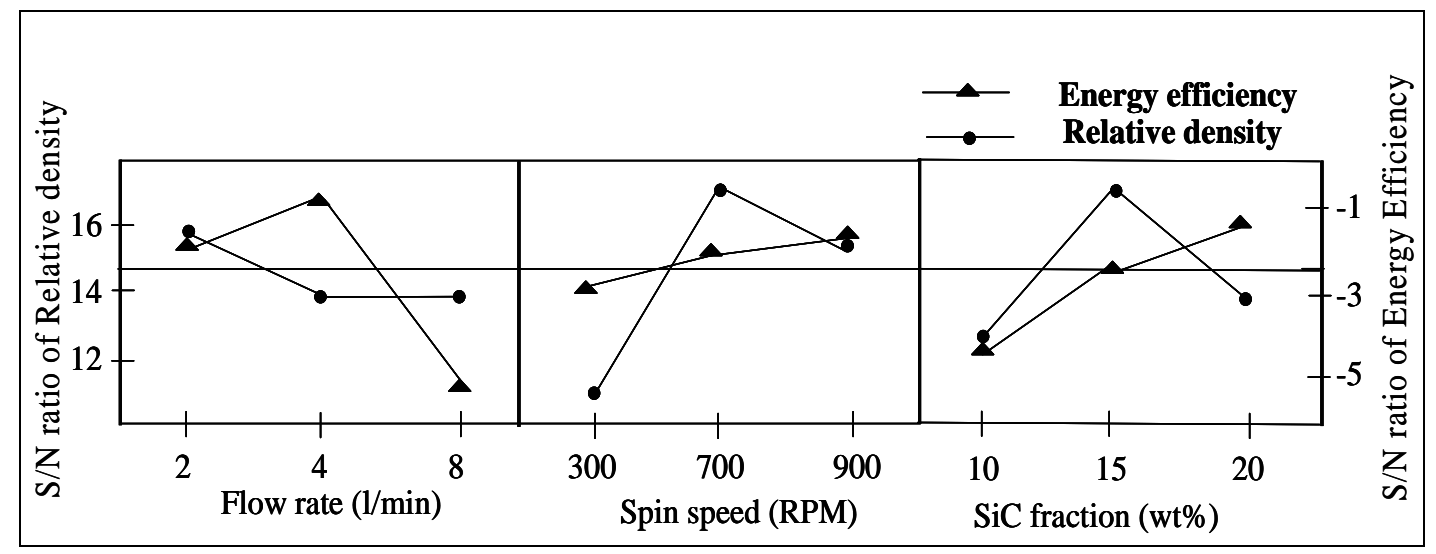

Figure 5: The mean $\mathrm{S} / \mathrm{N}$ ratio diagram of foaming parameters for relative density and energy efficiency.

\subsection{Multi-objective optimization of foam manufacturing process}

Regression analyses are performed on the data in Table III to get the relationship of the S/N ratios of the relative density and energy efficiency with the foaming parameters. The 
regression equations of the $\mathrm{S} / \mathrm{N}$ ratio of the relative density $(\mathrm{S} / \mathrm{N})_{\mathrm{RD}}$ and of the $\mathrm{S} / \mathrm{N}$ ratio of the energy efficiency $(\mathrm{S} / \mathrm{N})_{\mathrm{EE}}$ are expressed in the following form:

$$
\begin{aligned}
& (\mathrm{S} / \mathrm{N})_{\mathrm{RD}}=a_{0}+a_{1} Q+a_{2} N+a_{3} F+a_{4} Q N+a_{5} Q F+a_{6} N F+a_{7} Q^{2}+a_{8} N^{2}+a_{9} F^{2} \\
& (\mathrm{~S} / \mathrm{N})_{\mathrm{EE}}=b_{0}+b_{1} Q+b_{2} N+b_{3} F+b_{4} Q N+b_{5} Q F+b_{6} N F+b_{7} Q^{2}+b_{8} N^{2}+b_{9} F^{2}
\end{aligned}
$$

and the vectors of coefficients $\left[a_{0}, a_{1}, \ldots, a_{9}\right]$ and $\left[b_{0}, b_{1}, \ldots, b_{9}\right]$ are calculated by means of software Matlab.

The objective function for the optimization can now be formulated as the research of maximum of eq. 4 and 5 subjected to the operative window of process parameters:

$$
\begin{aligned}
2 & \leq Q \leq 8 \\
300 & \leq N \leq 900 \\
10 & \leq F \leq 20
\end{aligned}
$$

The solution of the objective functions leads to a combination of minimum relative density together with a maximum energy efficiency. The goal attainment method of Gembicki [21] is used to solve the presented multi-objective optimization problem. Applying this method, the equations (4) and (5) can be expressed in the following formulation:

Min: $\varepsilon$

$$
\text { Subject to: } \quad \begin{aligned}
(\mathrm{S} / \mathrm{N})_{\mathrm{RD}}-\omega_{1} \varepsilon & \leq(\mathrm{S} / \mathrm{N})^{\mathrm{T}}{ }_{\mathrm{RD}} \\
(\mathrm{S} / \mathrm{N})_{\mathrm{EE}}-\omega_{2} \varepsilon & \leq(\mathrm{S} / \mathrm{N})^{\mathrm{T}}{ }_{\mathrm{EE}} \\
2 & \leq Q \leq 8 \\
300 & \leq N \leq 900 \\
10 & \leq F \leq 20
\end{aligned}
$$

where $\varepsilon$ is an unrestricted scalar. $(\mathrm{S} / \mathrm{N})^{\mathrm{T}}{ }_{\mathrm{RD}}$ and $(\mathrm{S} / \mathrm{N})^{\mathrm{T}}{ }_{\mathrm{EE}}$ are the maximum $\mathrm{S} / \mathrm{N}$ ratios of the relative density and energy efficiency in Table III. Thus, they are the goals of the set of objectives $\left[(\mathrm{S} / \mathrm{N})_{\mathrm{RD}},(\mathrm{S} / \mathrm{N})_{\mathrm{EE}}\right]$. The weighting vector $\left[\omega_{1}, \omega_{2}\right]$ controls the relative degree of under or overachievement of the goals. In this paper, the weighting vector $\omega$ is set equal to the goal, so that the same percentage under-or-over attainment of the goals is achieved [21]. The solution of Eq. 6 gives the optimal combination of relative density and energy efficiency, as shown in Table IV, along with the estimated $\mathrm{S} / \mathrm{N}$ ratios of relative density and energy efficiency. From the Tables III-IV, it can be seen that this Pareto optimal combination of the manufacturing parameters comes as a trade-off between the two objectives.

Table IV: Optimal combination of parameters and estimated $\mathrm{S} / \mathrm{N}$ ratio.

\begin{tabular}{|c|c|c|c|c|}
\hline $\begin{array}{c}\text { Gas flow rate } \\
(\mathbf{l} / \mathbf{m i n})\end{array}$ & $\begin{array}{c}\text { Spin speed } \\
(\mathbf{R P M})\end{array}$ & $\begin{array}{c}\text { SiC content } \\
(\mathbf{w t} \%)\end{array}$ & $(\mathbf{S} / \mathbf{N})_{\mathbf{R D}}$ & $(\mathbf{S} / \mathbf{N})_{\mathbf{E E}}$ \\
\hline 2 & 633 & 16 & 20.045 & -0.273 \\
\hline
\end{tabular}

\subsection{Confirmation experiment}

In engineering analysis, a confirmation experiment is very important in validating the optimization problem and it is carried out at the optimum setting of the foaming parameters (Table IV). The obtained relative density is 0.103 and its $\mathrm{S} / \mathrm{N}$ ratio is 19.743 ; the obtained energy efficiency is 0.963 and its $\mathrm{S} / \mathrm{N}$ ratio is -0.327 . They show that both the relative density and energy efficiency are improved by using the optimal setting of the foaming parameters determined by the approach presented in this study. 


\subsection{Pareto set}

Pareto set of a problem is the collection of Pareto optimal solutions in a given multi-objective optimization problems. In this study, the weighting vector has been set equal to the goal, and a Pareto optimal combination of relative density and energy efficiency has been obtained. By varying the values of weight, a series of Pareto optimal combinations of both parameters will form the Pareto set for the foam manufacturing. Thus, a series of values between 0 and 1 are chosen for the weight $\omega_{1}$, and the weight $\omega_{2}$ is set equal to $1-\omega_{1}$. Based on this consideration, the Pareto set is determined by solving the equation of goal attainment method (eq. 6). The corresponding spin speed and SiC fraction are determined and plotted in Fig. 6, while the best flow rate is $2 \mathrm{l} / \mathrm{min}$ for each solution. If a specific foaming parameter is requested, by the plot of Fig. 6 it is possible to obtain the corresponding optimal other parameter.

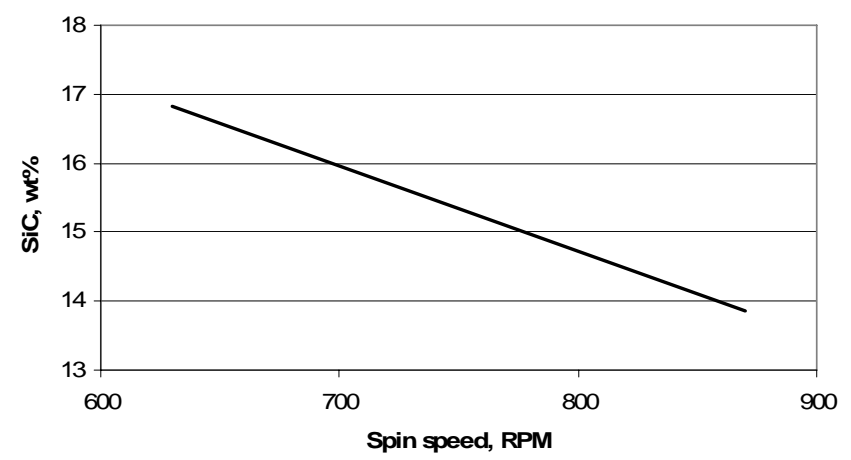

Figure 6: Pareto optimal combinations of spin speed and SiC fraction.

\section{CONCLUSIONS}

In this paper a new method of optimization of the manufacturing parameters for foam production has been suggested. In fact, the optimization with regard to multiple objective functions, aiming at a simultaneous improvement of the objectives, is usually difficult; the goals are conflicting so that an optimal solution in the conventional sense does not exist. By using in integrated manner the Taguchi method (the orthogonal array of $\mathrm{L}_{9}$ and $\mathrm{S} / \mathrm{N}$ ratio) and the goal attainment method, a systematic and efficient methodology was provided.

In the light of this analysis the following conclusions can be drawn:

- the significant foaming parameters affecting the process can be identified by performing the experiments designed to the orthogonal array of the Taguchi method saving time and costs;

- the relative density and energy efficiency are improved simultaneously through the multiobjective optimization approach;

- the optimal foaming parameters for relative density and energy efficiency in MGI process are: gas flow rate at $2 \mathrm{l} / \mathrm{min}$, spin speed at $633 \mathrm{RPM}$ and SiC fraction at $16 \mathrm{wt} \%$;

- the corresponding Pareto set has been found and it can be useful to the user for choosing a qualified solution for the manufacturing problems.

As a global result, Taguchi method and the goal attainment method are very power tools to solve the multi-objective optimization of foam manufacturing.

\section{ACKNOWLEDGEMENT}

Financial support from research project PRIN 06 - TILAS (Innovative Manufacturing Technologies for Metal Foams) is acknowledged. 


\section{REFERENCES}

[1] Wood, J. T. (1997). Production and applications of continuously cast, foamed aluminium, Proceedings of Fraunhofer USA Metal Foam Symposium, Verlag MIT Publishing, 31-35

[2] Asholt, P. (1999). Aluminium foam produced by the melt foaming route process, properties and applications, Proceedings of Int. Conf. on Metal Foam and Porous Metal Structures, MIT Publishing, 133-140

[3] Ip, S. W.; Wang, Y.; Tuguri, J. M. (1999). Aluminium foam stabilization by solid particles, Canadian Metallurgical Quarterly, Vol. 38, No. 1, 81-92

[4] Kaptay, G. (2004). Interfacial criteria for stabilization of liquid foams by solid particles, Colloids and Surfaces A: Physicochemical and Engineering Aspects, Vol. 230, 67-80

[5] Hur, B. Y.; Park, S. H.; Hiroshi, A. (2003). Viscosity and surface tension of Al and effects of additional elements, Materials Science Forum, Vol. 439, 51-56

[6] Babcsan, N.; Leitlmeier, D.; Degischer, H. P. (2003). Foamability of particle reinforced aluminium melt, Mat.-wiss. u. Werkstofftech, Vol. 34, 22-29

[7] Leitlmeier, D.; Degischer, H. P.; Flankl, H. J. (2002). Development of a foaming process for particulate reinforced aluminium melts, Advanced Engineering Materials, Vol. 4, No. 10, 735740

[8] Deqing, W.; Ziyuan, S. (2003). Effect of ceramic particles on cell size and wall thickness of aluminium foam, Materials Science \& Engineering A, Vol. 361, 45-49

[9] Taguchi, G. (1990). Introduction to quality engineering, McGraw-Hill, New York

[10] Nalbant, M.; Gokkaya, H.; Sur, G. (2007). Application of Taguchi method in the optimization of cutting parameters for surface roughness in turning, Materials \& Design, Vol. 28, No. 4, 13791385

[11] Yang, W. H.; Tarng, Y. S. (1998). Design optimization of cutting parameters for turning operations based on the Taguchi method, Journal of Materials Processing Technology, Vol. 84, $122-129$

[12] Ghani, J. A.; Choudhury, I. A.; Hassan, H. H. (2003). Application of Taguchi method in the optimization of end milling parameters, Journal of Materials Processing Technology, Vol. 145, No. 1, 84-92

[13] Vijian, P.; Arunachalam, V. P. (2006). Optimization of squeeze cast parameters of LM6 aluminium alloy for surface roughness using Taguchi method, Journal of Materials Processing Technology, Vol. 180, No. 1-3, 161-166

[14] Olabi, A. G.; Casalino, G.; Benyounis, K. Y.; Hashmi, M. S. J. (2006). An ANN and Taguchi algorithms integrated approach to the optimization of $\mathrm{CO} 2$ laser welding, Advances in engineering software, Vol. 37, No. 10, 643-648

[15] Ko, D.-C.; Kim, D.-H.; Kim, B.-M. (1999). Application of artificial neural network and Taguchi method to preform design in metal forming considering workability, International journal of machine tools \& manufacture, Vol. 39, No. 5, 771-785

[16] Yang, H.-J.; Hwang, P.-J.; Lee, S.-H. (2002). A study of shrinkage compensation of the SLS process by using the Taguchi method, International journal of machine tools \& manufacture, Vol. 42, No. 11, 1203-1212

[17] Shabeer, S.; Wang, M. Y. (2000). Multi-objective optimization of sequential brakeforming processes, Journal of Materials Processing Technology, Vol. 102, 266-276

[18] Jaishi, B.; Ren, W. X. (2007). Finite element model updating based on eigenvalue and strain energy residuals using multiobjective optimisation technique, Mechanical systems and signal processing, Vol. 21, No. 5, 2295-2317

[19] Benouali, A.-H.; Froyen, L.; Delerue, J. F.; Wevers, M. (2002). Mechanical analysis and microstructural characterisation of metal foams, Materials Science and Technology, Vol. 18, No. 5, 489-494

[20] Cymat Corp. (2002). Standard test method for compressive properties of metal foams, Technical manual for stabilized aluminium foam, Ontario, Canada, pp. A5-1

[21] Gembicki, F. W. (1974). Vector Optimization for Control with Performance and Parameter Sensitivity Indices, Ph.D. Thesis, Case Western Reserve Univ., Cleveland, Ohio 